\title{
Headache as a First Manifestation of Vogt-Koyanagi-Harada Disease
}

\author{
Katerina K. Kosma ${ }^{a}$ Evangelia Kararizou $^{a}$ Ioannis Markou ${ }^{b}$ \\ Evanthia Eforakopoulou $^{\mathrm{b}}$ Grigoris Kararizos ${ }^{\mathrm{b}}$ Charalampos Mitsonis $^{\mathrm{a}}$ \\ Konstantinos Gkiatas ${ }^{b}$ \\ ${ }^{a}$ Department of Neurology, University of Athens, Aeginition Hospital, and ${ }^{\mathrm{b}} 251$ Hellenic Air Force General Hospital, \\ Athens, Greece
}

\section{Key Words}

Vogt-Koyanagi-Harada disease $\cdot$ Headache $\cdot$ Uveitis

\begin{abstract}
Objectives: To describe headache as an initial presentation of Vogt-Koyanagi-Harada (VKH) disease. Clinical Presentation and Intervention: A 56-year-old man initially presented with a 6-month duration of a continuous, diffuse headache of mild to moderate and sometimes of severe intensity. A CT of the brain was normal. Neurological examination was normal, including absence of symptoms and signs of meningismus. During the last 2 months, an intermittent eye pain, redness, and gradual loss of vision in both eyes was detected and subsequently he had tinnitus, malaise, nausea and mild meningismus. He was diagnosed as having VKH disease. Ophthalmologic examination revealed high intraocular pressure, requiring trabeculectomy with surgical iridectomy. The patient is now being treated with systemic steroids. Conclusion: VKH disease may initially present as sustained headache, without specific ophthalmologic symptoms and it should be considered in the differential diagnosis in patients with atypical but relentless headache.
\end{abstract}

Copyright $\odot 2008$ S. Karger AG, Basel

\section{Introduction}

Vogt-Koyanagi-Harada (VKH) disease is an uncommon granulomatous inflammatory disorder affecting the eyes, ears, meninges, brain and skin $[1,2]$. Although the etiology of VKH disease is unknown, clinical and histopathologic evidence support an autoimmune reaction directed against antigens shared by uveal, dermal, and meningeal melanocytes [3-5]. The diagnostic criteria are based on the concept that VKH disease is a single entity with very different clinical manifestations depending upon the stage at which the patient is examined [6]. We report a case of a patient with a 6-month history of sustained undiagnosed headache as a first symptom of $\mathrm{VKH}$ disease. The characteristic ophthalmologic findings of the disease developed late in the course.

\section{Case Report}

A 56-year-old man presented with a 6-month history of sustained undiagnosed headache. The pain was described as continuous, diffuse and throbbing. It was mild to moderate and sometimes of severe intensity. During the last 3 months, it was associated with nausea. Sometimes the pain was located behind

\section{KARGER}

Fax +4161306 1234

E-Mail karger@karger.ch

www.karger.com
(C) 2008 S. Karger AG, Basel

1011-7571/08/0173-0253\$24.50/0

Accessible online at:

www.karger.com/mpp
Dr. Evangelia Kararizou

Neurologic Clinic

Aeginition Hospital, 72-74, Vass. Sofias Ave.

GR-11528 Athens (Greece)

Tel. +30 2107289 115, Fax +30 2107216 474, E-Mail ekarariz@med.uoa.gr 
one eye or in the eye region, but the affected eye was not swollen and the conjunctiva was not red. Other associated symptoms, such as vomiting, photophobia, sensitivity to sound or tearing were not reported. A CT of the brain was normal. Neurological examination was normal, including absence of signs of meningismus. He had digital clubbing.

Six months after the onset of the headache and during the last 2 months the patient reported intermittent eye pain and redness and gradual loss of vision in both eyes. Best-corrected visual acuity was $3 / 10$ in the right eye and $4 / 10$ in the left eye. Intraocular pressures were normal. Slit-lamp examination of the anterior segment showed bilateral granulomatous keratic precipitates on the inferior corneal endothelium and severe anterior chamber cell and flare and mild vitritis bilaterally. Fundus examination was normal. Laboratory investigations were negative. The patient was treated initially with topical corticosteroids and cycloplegics, with marked improvement in intraocular inflammation. However, intraocular pressures increased to $30-40 \mathrm{~mm} \mathrm{Hg}$, requiring topical antiglaucoma medication. While tapering treatment with local steroids the uveitis relapsed and treatment was reintroduced at the initial dose.

Eight months after the onset of symptoms, he had tinnitus, headache and nausea. The patient, being an Air Force officer, was transferred to the Air Force Hospital. Neurological examination revealed mild meningismus but cerebrospinal fluid examination was normal. Ophthalmologic examination revealed high intraocular pressures, requiring trabeculectomy with surgical iridectomy. Antiglaucoma therapy was stopped and visualization of the fundus after surgery disclosed widespread atrophy of the retinal pigment epithelium and focal areas of subretinal fluid. The patient is now being treated with systemic steroids. Four months later, reexamination showed that his visual acuity remains low due to cataract formation in both eyes.

The revised diagnostic criteria for VKH disease has three divisions: complete, incomplete and probable VKH [6]. Our patient fulfils the criteria for the incomplete type of the disease since he had no history of penetrating ocular trauma or surgery; no laboratory or clinical evidence suggestive of other ocular disease; ocular involvement with focal areas of subretinal fluid and recurrent areas of anterior uveitis was bilateral and he also had neurological and auditory findings. Our patient did not have any integumentary finding such as vitiligo or poliosis. However, these findings usually appear later in the course of the disease.

\section{Discussion}

VKH disease is a multisystem disorder. The diagnosis requires evidence of involvement of more than the ocular system $[1,7]$. However, patients presenting early with this disease may complain of auditory or neurologic manifestations (such as tinnitus, dysacusis, nausea, vertigo, meningismus) and visual loss, exudative retinal detachments and optic disk hyperemia may develop later.

Current diagnostic criteria are based on the concept that $\mathrm{VKH}$ disease is a single entity with different clinical manifestations depending on the stage at which the patient is examined. The criteria consist of five sections [6] and are designed to allow for division of 'definite' VKH disease into 'complete' and 'incomplete' categories based on the spectrum of manifestations seen and both have an absolute requirement for the presence of bilateral ocular involvement. When the diagnosis is not certain, but the findings are highly suggestive, the proposed new criteria allow a designation of 'probable' VKH disease, wherein the ocular and exclusionary criteria are met, but the nonocular criteria are not met $[1,6]$. Our patient fulfils the criteria for the incomplete type of the disease.

Once diagnosed, multiple therapeutic regimens have been used in the treatment of VKH disease, including observation, local, oral, and intravenous corticosteroids, cyclosporine, antimetabolites, and alkylating agents $[1,7]$.

\section{Conclusion}

We emphasize that VKH disease may initially present as sustained headache, without specific ophthalmologic symptoms, which should be considered in the differential diagnosis in patients with atypical but relentless headache.

\section{References}

1 Andreoli CM, Foster CS: Vogt-KoyanagiHarada disease. Int Ophthalmol Clin 2006; 46:111-122.

-2 Beniz J, Forster DJ, Lean JS, Smith RE, Rao NA: Variations in clinical features of the Vogt-Koyanagi-Harada syndrome. Retina 1991;11:275-280.

3 Svitra PP, Perry H: Vogt-Koyanagi-Harada (uveomeningitic) syndrome; in Albert DM, Jakobiec FA (eds): Principles and Practice of Ophthalmology: Clinical Practice. Philadelphia, WB Saunders, 1994, vol 1, chapt 31, pp 481-488.
-4 Nomura S, Matsuzaki T, Ozaki Y, Yamaoka M, Yoshimura C, Katsura K, Xie GL, Kagawa H, Ishida T, Fukuhara S: Clinical significance of HLA-DRB1*0410 in Japanese patients with idiopathic thrombocytopenic purpura. Blood 1998;91:3616-3622.

5 Yamaki K, Gocho K, Sakuragi S: Pathogenesis of Vogt-Koyanagi-Harada disease. Int Ophthalmol Clin 2002;42:13-23.
-6 Read RW, Holland GN, Rao NA, Tabbara KF Ohno S, Arellanes-Garcia L, Pivetti-Pezzi P, Tessler HH, Usui M: Revised diagnostic criteria for Vogt-Koyanagi-Harada disease: report of an international committee on nomenclature. Am J Ophthalmol 2001;131: 647-652.

7 Moorthy RS, Inomata H, Rao NA: VogtKoyanagi-Harada syndrome. Surv Ophthalmol 1995;39:265-292. 